\title{
Two differential flows in a bioreactor promoted platelet generation from human pluripotent stem cell-derived megakaryocytes.
}

\section{$\operatorname{AUTHOR}(\mathrm{S}):$}

Nakagawa, Yosuke; Nakamura, Sou; Nakajima, Masahiro; Endo, Hiroshi; Dohda, Takeaki; Takayama, Naoya; Nakauchi, Hiromitsu; Arai, Fumihito; Fukuda, Toshio; Eto, Koji

\section{CITATION:}

Nakagawa, Yosuke ... [et al]. Two differential flows in a bioreactor promoted platelet generation from human pluripotent stem cell-derived megakaryocytes.. Experimental hematology 2013, 41(8): 742-748

\section{ISSUE DATE:}

2013-08

\section{URL:}

http://hdl.handle.net/2433/177915

\section{RIGHT:}

(C) 2013 ISEH - Society for Hematology and Stem Cells. Published by Elsevier Inc.; This is not the published version. Please cite only the published version.; この論文は出版社版でありません。引用の際には 出版社版をご確認ご利用ください。 


\title{
Two differential flows in a bioreactor promoted platelet generation from human pluripotent stem cell-derived megakaryocytes
}

\author{
Yosuke Nakagawa $^{1,5}$, Sou Nakamura ${ }^{2}$, Masahiro Nakajima ${ }^{1,3}$, \\ Hiroshi Endo ${ }^{2}$, Takeaki Dohda ${ }^{2}$, Naoya Takayama ${ }^{2}$, \\ Hiromitsu Nakauchi ${ }^{4}$, Fumihito Arai ${ }^{1}$, Toshio Fukuda ${ }^{1,3,5}$, Koji Eto ${ }^{2,4}$
}

${ }^{1}$ Department of Micro-Nano Systems Engineering, Nagoya University, Aichi 464-8603 Japan. ${ }^{2}$ Clinical Application Department, Center for iPS Cell Research and Application, Kyoto University, 606-8507, Japan. ${ }^{3}$ Center For Micro-nano Mechatronics, Nagoya University, Aichi 464-8603, Japan. ${ }^{4}$ Laboratory of Stem Cell Therapy, Center for Stem Cell Biology and Regenerative Medicine, The Institute of Medical Science, The University of Tokyo, Tokyo 108-8639, Japan

${ }^{5}$ These authors contributed equally to this work

\section{Contact to}

Dr. Koji Eto, MD, PhD

Clinical Application Department, Center for iPS Cell Research and Application, Kyoto University, 53 Kawahara-cho, Shogoin, Sakyo-ku, Kyoto 606-8507, Japan.

Tel; +81-075-366-7075 Fax; +81-075-366-7095

Email: kojieto@cira.kyoto-u.ac.jp

\section{Abstract, 209 words; Main text, 2083 words, 6 figures, 1 table}

Key word: platelet, megakaryocyte, bioreactor, device, flow, shear stress 


\section{Abstract (209 words)}

Induced pluripotent stem cell (iPSC) technology enables us to investigate various potential iPSC-based therapies. Although the safety of iPSC-derivation has not been completely validated, anucleate cells, such as platelets or erythrocytes, derived from iPSCs are promising targets. However, the efficiency of in vitro platelet generation from megakaryocytes (MKs) under static culture conditions is lower than is seen in vivo. Here we demonstrate the proof-of-concept by a two-dimensional flow culture system that enabled us to increase platelet yield from human embryonic stem cell (hESCs) or iPSC-derived MKs using a biomimetic artificial blood vessel system. The bioreactor was composed of biodegradable scaffolds with ordered arrays of pores made to mimic in vivo bone marrow through salt-leaching. Within the system, two flows in different directions in which the angle between the directions of flow is $60^{\circ}$ but not $90^{\circ}$ contributed to suitable pressure and shear stress applied to MKs to promote platelet generation. Generated platelets derived from hESCs or hiPSCs through the bioreactor with $60^{\circ}$ angle revealed intact integrin $\alpha \operatorname{IIb} \beta 3$ activation after agonist stimulation. Collectively, our findings indicate that two flows in different directions of two-dimensional flow culture may be a feasible system for in vitro generation of platelets from pluripotent stem cells, i.e. iPSC-derived MKs, in numbers sufficient for transfusion therapy. 


\section{Introduction}

Platelets are essential for hemostasis and thrombosis, and also play important roles in wound repair, inflammatory reactions, angiogenesis, lymph-vessel separation, and tumor metastasis (1). In humans, approximately $1 \times 10^{11}$ platelets are produced each day, presumably through the cytoplasmic fragmentation of megakaryocytes (MKs) and/or formation of proplatelets, elongated pseudopods that extend from mature MKs and release platelets (2). Platelets production requires the commitment of hematopoietic stem cells (HSCs)/hematopoietic progenitor cells (HPCs) to the MK lineage, followed by proliferation of the progenitors and terminal differentiation. Ultimately, immature MKs develop a unique membrane complex called the demarcation membrane (DM) system, which is thought to serve as a membrane reservoir for platelet biogenesis through both direct cytoplasmic rupture and elongation of proplatelets (3). Both of those processes are controlled by the orchestrated activities of numerous signaling molecules (3). A variety of gene-targeted mouse models have shed light on the roles played by the transcriptional factors involved in platelet formation, and have contributed to the characterization of the linkage between the machinery of MK maturation and platelet release $(3,4,5,6,7)$. However, the actual machinery of platelet release (platelet biogenesis) from MKs has not yet been characterized (8).

The establishment of mouse (9) and human embryonic stem cells (hESCs) (10) and human induced pluripotent stem cells (hiPSCs) $(11,12)$ were crucial advances in the area of regenerative medicine. Notably, hiPSC-based platelet generation is a particularly fascinating therapy model, in part because blood donors are becoming increasing scarce in most industrial countries, including Japan (13). In addition, because platelets are anucleate, they can be 
subjected to gamma irradiation prior to their transfusion, which would prevent the occurrence of oncogenesis or teratoma formation due to the presence of residual undifferentiated hiPSCs or hiPSC-derived cells. Finally, for patients requiring repeated transfusions, it is important that the platelets are human leukocyte antigen (HLA)-matched (13); thus an HLA-identical donor could provide the origin cells for creating iPSCs that contribute to a sustained platelet supply. Here, by primarily using human ESCs as a gold standard of reference $(14,15)$, we describe a two-dimensional flow culture that recapitulates bone marrow in the view of "blood flow" application and may be a feasible system for in vitro generation of platelets from human pluripotent stem cell-derived MKs in numbers sufficient for transfusion therapy. 


\section{Methods}

\section{Cells and Reagents}

The KhES-3 human ESC clone (Institute for Frontier Medical Science, Kyoto University, Kyoto, Japan) was used upon the approval by the Minister of Education, Culture, Sports, Science, and Technology of Japan (MEXT) (14). In addition, The TkDA3-4 hiPSC clone was used (15). The mouse C3H10T1/2 cell line was purchased from the RIKEN Bio-Resource Center (Tsukuba, Ibaraki, Japan) and was cultured as described previously $(14,15)$. Human umbilical cord endothelial cells (HUVECs) were purchased from KURABO (Tokyo, Japan). Human vascular endothelial growth factor (VEGF), human thrombopoietin (TPO) and human stem cell factor (SCF) were all from R\&D Systems (Minneapolis, MN). Heparin was from Ajinomoto Pharmaceuticals Co., LTD (Tokyo, Japan). The following antibodies and stains were obtained from BD Biosciences (Franklin Lakes, NJ): allophycocyanin (APC)-conjugated anti-CD41a, FITC-conjugated anti-CD41a (GPIX), anti-CD42b (GPIb $\alpha$ ) and Hoechst 33342 (blue). DAPI (Vector Laboratories, Burlingame, CA) and Hoechst 33342 were used for nuclear staining in MKs. FITC-conjugated PAC-1 antibody (BD Biosciences, San Diego, CA) was used for platelet activation studies as described $(14,15)$.

\section{Hematopoietic differentiation of human ESCs or iPSCs}

Differentiation of human ESCs or iPSCs into hematopoietic cells was performed as described previously $(14,15)$. In brief, small clumps of ESCs or iPSCs $(<100$ cells treated with PBS containing $0.25 \%$ trypsin (Invitrogen, Carlsbad, CA), $1 \mathrm{mM} \mathrm{CaCl}_{2}$ (Sigma-Aldrich, St. Louis, 
MI) and 20\% Knock-out Serum Replacement (KSR, Invitrogen [Life technology Japan, Tokyo]) were transferred onto mitomycin (Sigma)-treated or irradiated C3H10T1/2 cells and co-cultured in hematopoietic cell differentiation medium (Iscove's Modified Dulbecco's Medium [IMDM, Life technology Japan, Tokyo] with 15\% fetal bovine serum and VEGF as described previously $(14,15)$, which was replaced every 3 days. On days $14-15$ of culture, the HPCs within ES(14) or iPS-Sacs (15) were collected, transferred onto fresh mitomycin-treated or irradiated feeder cells, and further cultivated in differentiation medium supplemented with $50 \mathrm{ng} / \mathrm{ml}$ TPO, $50 \mathrm{ng} / \mathrm{ml} \mathrm{SCF}$ and $25 \mathrm{U} / \mathrm{ml}$ heparin sodium, as described $(14,15)$. The medium was refreshed every 3 days, and non-adherent cells were collected on day 20 of culture and transferred to a bioreactor.

\section{Flow cytometric analysis of platelets}

Washed platelets were prepared as described previously $(14,15)$. The resultant platelet pellets were resuspended with staining medium and stained with anti-human CD41a-APC and CD42b-PE for $30 \mathrm{~min}$ at room temperature. The platelets were then diluted in $200 \mu \mathrm{l}$ of staining medium and analyzed by flow cytometry (FACSAria, BD biosciences, San Diego, CA). Platelet numbers were counted using True Count Beads (BD Biosciences).

\section{Construction of a bioreactor to obtain platelets from human iPSC-derived MKs}

The fabrication of a bioreactor to produce platelets entailed the following steps. Using photo-resist SU8-3050 (Nippon Kayaku, Tokyo, Japan), a microfluidic device was constructed by centrifugation on a silicon wafer at $5000 \mathrm{rpm}$ for $30 \mathrm{~s}$. The device was then exposed after 
applying a mask using a mask aligner, developed in developer and rinsed with de-ionized water. Polydimethylpolysiloxane (PDMS) was then solidified on the fabricated mold at $60^{\circ} \mathrm{C}$ in an oven. Finally, the PDMS was released from the mold and bonded to a glass plate.

\section{Experimental procedure (dissemination of cells, culture and counting platelets)}

Sterilization of the bioreactor was achieved through treatment with ethanol for $30 \mathrm{~min}$ irradiation with UV light. Platelet generation in the bioreactor was observed in a connected circulation system on the stage of a confocal microscope (FV1000, Olympus, Tokyo, Japan) at $37^{\circ} \mathrm{C}$. The process of platelet generation from MKs was visualized using a confocal microscope system, and the images were recorded in the hard disc of a connected computer. Flow pressure was determined by flow speed and the medium collected, including the cell populations in the reservoir. After adding ACD medium to the culture medium (1:10 dilution), the collected samples were centrifuged at $900 \mathrm{rpm}$ for $10 \mathrm{~min}$ (slow acceleration). Finally, the cell samples were stained for cell surface molecules specific for MKs/platelets and analyzed by flow cytometry.

\section{$\underline{\text { Statistics }}$}

We evaluated results by using an unpaired t-test for significance. 


\section{Results and Discussion}

Observations in living mice made using two-photon confocal microscopy revealed that platelet release occurs within both the capillaries and sinusoids of the bone marrow (8). Within human bone marrow, sinusoidal capillaries are the discontinuous type, with wall thicknesses less than $30 \mu \mathrm{m}$ and containing numerous pores. One approach to reconstituting the structure of such vessels in vitro is to use a sheet-like scaffold with multiple pores (16). We constructed a structure using the salt-leaching method as shown in Figure 1A. The contents of the solution used for the salt-leaching are shown in Table 1.

Using the constructed sheet-like porous structure, we designed the bioreactor to recapitulate mainly two functions of capillary blood vessel for platelet production: (i) one function that is a porous structure of endothelial cells for fixing MKs on the outside of blood vessel wall (17), and, (ii) another function that is a platelet production of MKs by applying the shearing effects of blood flow. Within the bioreactor, the membrane scaffold for platelet release from MKs was composed of PDMS. Moreover, the scaffold was completely covered by HUVECs where adjacent chamber with a layer of HUVECs and a formed porous barrier in between appeared $30 \mu \mathrm{m}$. By adjusting the speed of flow through the structure, different levels of shear stress were generated, which were calculated using equation (A), where $Q$ is flow speed $[\mathrm{ml} / \mathrm{h}], \mu$ is the viscosity of the medium $[\mathrm{Pa} / \mathrm{s}], a$ is the width of the duct $[5.0 \mathrm{~mm}]$, and $b$ is the height of duct $[0.3 \mathrm{~mm}]$.

$$
\tau=\mu \frac{6 Q}{a b^{2}}
$$


In the system, the medium flows past MKs on HUVECs in two directions (pressure flow and main flow), such that the pressure flow is perpendicular to the main flow; the main flow applies shear stress to the MKs, while the pressure flow applies pressure for MK fixation to the each slit. Thus we estimated that exposed shear stress was occurred from $0.14-37.5 \mathrm{dyne} / \mathrm{cm}^{2}$ onto MKs within chamber and iPSC-derived MKs were applied into the regions of the pores, as shown in the left panel of Figure 1B, although it is not ruled out that the flow was turbulence.

In addition, we expected that vascular cell adhesion molecule-1 (VCAM-1) on HUVEC might promote platelet release from MKs because of the reports that integrin $\alpha 4 \beta 1$ expression in MKs is crucial for platelet production in vivo in mouse models (reference 18) or that the binding between integrin $\alpha 4 \beta 1$ in MKs and VCAM-1 promoted proplatelets, a hallmark of platelet generation, from cultured MKs in vitro (19).

Our bioreactor system enabled us to visualize real-time changes to MKs during culture. Time-lapse confocal microscopy revealed that applied iPSC-derived MKs grew on HUVECs within the bioreactor (Figure 2A), and the numbers of adherent MKs were greater under weak flow conditions $\left(0.14 \mathrm{dyne} / \mathrm{cm}^{2}\right.$ ) (Figure 2B). We applied $1.2 \times 10^{5} \mathrm{MKs}$ totally into the

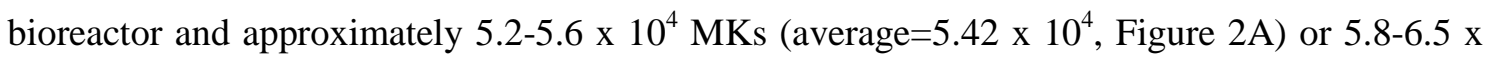
$10^{4}$ MKs (average $=6.2 \times 10^{4}$, Figure 2B) were counted in the absence or presence of flow respectively. When we compared the final yield of platelets between the static culture (no flow) in a conventional culture dish (14) and our new bioreactor system with flow, as shown in Figure 3A, we found that platelet generation from human ESC-derived MKs tended to be better in the flow system (Figure 3B). Moreover, time-lapse imaging of the platelet production from MKs under flow revealed that increasing the shear stress by adjusting flow speed $(1.0 \mathrm{ml} / \mathrm{hr})$ 
stimulated large-proplatelet (90 min in Figure 4), followed by cytoplasmic fragmentation from ESC-derived MKs (92 min in Figure 4). However, the increase in platelet production was not significant (Figure 3B).

To improve platelet yield, we designed a new bioreactor using two flows with an angle between the main and pressure flows. The schematic in Figure 5A shows the newer design of a bioreactor, in which the angle between the directions of flow is $60^{\circ}$. The platelets were generated from MKs placed between the slits. We again applied $1.2 \times 10^{5}$ MKs for each experiment within a newer bioreactor. Under these conditions, the numbers of platelets produced from human ESCs were as much as 3.6 times higher than in static culture (mean count: 19822 under static vs. 71755 under flow, Figure 5B, from independent 4 experiments, $\mathrm{p}<0.05)$. These results using the same improved reactor system were confirmed by using human iPSC-derived MKs (2 experiments, data not shown). Purity of $\mathrm{CD}^{4} 1 \mathrm{a}^{+}$or $\mathrm{CD} 42 \mathrm{~b}^{+}$platelet populations was also examined by flow cytometer and there was no significant difference between static condition and bioreactor (Figure 5C and 5D).

In order to confirm the functionality by collected platelets through second generation of a bioreactor in Figure 5A, agonist-induced integrin activation in platelets was examined. The PAC-1 binding, the activated form of an integrin $\alpha \operatorname{Ilb} \beta 3$ after agonist stimulation showed intact functionality by hESC- or iPSC-derived platelets (Figure 6), confirming the similarity to the previous studies obtained under static conditions $(14,15)$.

Using a novel 3-dimensional bioreactor, which enabled us to regulate shear stress through application of flow in two directions, we enhanced platelet generation from MKs by subjecting the cells to the appropriate levels of shear stress. Within this system the shear stress created by 
the main flow could be regulated by the pressure flow. Furthermore, it appears that an angle of $60^{\circ}$ between the main and pressure flows is optimal.

This novel conception has the potential to influence a new era of regenerative medicine through the use of hESCs or hiPSCs. In an effort to better understand the underlying molecular mechanisms of platelet release and production from MKs, we are attempting to mimic previously visualized in vivo platelet production (8), which showed evidence of shear stress dependence. Recently, several groups reported in vitro platelet generation under conditions in which high shear stress was produced utilizing one-directional flow $(18,21)$. On the other hand, our proposed bioreactor makes use of flow in two directions. We suggest that the flow applying pressure to the cells stimulates MK maturation on HUVECs, while the flow applying shear stress stimulates platelet generation. In addition, it appears that the angle between those two-directional flow a key for improving production efficiency as exemplified by three times difference in comparison between Figures 3 and 5). This two-direction flow strategy is seemingly a feasible approach to the future development of bioreactors able to supply platelets derived from human pluripotent stem cells in numbers sufficient for transfusion. 


\section{References}

1. Leslie M. Cell biology. Beyond clotting: the powers of platelets. Science. 328(5978):562-564, 2010.

2. Kaushansky K. Historical review: megakaryopoiesis and thrombopoiesis. Blood. 11(3):981-986, 2008.

3. Patel SR, Hartwig JH, Italiano JE Jr. The biogenesis of platelets from megakaryocyte proplatelets. J Clin Invest. 115(12):3348-3354, 2005.

4. Shivdasani RA. Transcription factor NF-E2 is required for platelet formation independent of the actions of thrombopoeitin/MGDF in megakaryocyte development. Cell. 81(5):695-704, 1995.

5. Pevny L, Lin CS, D'Agati V, et al. Development of hematopoietic cells lacking transcription factor GATA-1. Development. 121(1):163-172, 1995.

6. Tsang AP, Visvader JE, Turner CA, et al. FOG, a Multitype Zinc Finger Protein, Acts as a Cofactor for Transcription Factor GATA-1 in Erythroid and Megakaryocytic Differentiation. Cell.90(1):109-119, 1997.

7. Ware J, Russell S, Ruggeri ZM. Generation and rescue of a murine model of platelet dysfunction: The Bernard-Soulier syndrome. Proc Natl Acad Sci USA. 97(6):2803-2808, 2000. 8. Junt T, Schulze H, Chen Z, et al. Dynamic visualization of thrombopoiesis within bone marrow. Science. 317(5845):1767-1770, 2007.

9. Evans MJ, Kaufman MH. Establishment in culture of pluripotential cells from mouse embryos. Nature. 292(5819):154-156, 1981. 
10. Thomson JA. Embryonic stem cell lines derived from human blastocysts. Science. 282(5391):1145-1147, 1998.

11. Takahashi K, Yamanaka S. Induction of pluripotent stem cells from mouse embryonic and adult fibroblast cultures by defined factors. Cell. 126(4):663-676, 2006.

12.Takahashi K, Tanabe K, Ohnuki M, et al. Induction of Pluripotent Stem Cells from Adult Human Fibroblasts by Defined Factors. Cell. 131(5):861-872, 2007.

13. Stroncek DF, Rebulla P. Platelet transfusions. Lancet. 370(9585): 427-38, 2007.

14. Takayama N, Nishikii H, Usui J, et al: Generation of functional platelets from human embryonic stem cells in vitro via ES-sacs, VEGF-promoted structures that concentrate hematopoietic progenitors. Blood 111:5298-5306, 2008.

15. Takayama N, Nishimura S, Nakamura S, et al: Transient activation of c-MYC expression is critical for efficient platelet generation from human induced pluripotent stem cells. J Exp Med 207:2817-2830, 2010

16. Uchida, T, Oura $\mathrm{H}$., et al. Development of biodegradable scaffolds by leaching self-assembled magnetic sugar particles, Proceedings of IEEE International Symposium on Micro-Nano Mehatronics and Human Science in 2007, 356-361, 2007.

17. Milsky, I. The effect of age on the wall stiffness of the human thoracic aorta: a large deformation "anisotropic" elastic analysis., Journal of Theoretical Biology, 59(2): 467-484, 1976. 
18. Avecilla ST, Hattori K, Heissig B, et al. Chemokine-mediated interaction of hematopoietic progenitors with the bone marrow vascular niche is required for thrombopoiesis. Nat Med. 10:64-71, 2004.

19. Takizawa H, Eto K, Yoshikawa A, et al. Growth and maturation of megakaryocytes is regulated by Lnk/Sh2b3 adaptor protein through crosstalk between cytokine- and integrin-mediated signals. Exp Hematol. 36(7):897-906, 2008.

20. Dunois-Lardé C, Capron C, Fichelson S et al. Exosure of human megakaryocytes to high shear rates accelerates platelet production. Blood. 114:1875-1883, 2009.

21. Pallotta I, Lovett M, Kaplan DL, Balduini A. Three-dimensional system for the in vitro study of megakaryocytes and functional platelet production using silk-based vascular tubes.

Tissue Eng Part C Methods. 17(12):1223-32, 2011.

\section{Acknowledgements}

The authors thank Drs. N. Nakatsuji and H. Suemori (Institute for Frontier Medical Sciences, Kyoto University) for providing human KhES-3 cell lines. This work was supported by New Research Area Promotion Grant "Bio Assembler" from MEXT, Japan (F.A., H.N., T.F., and K.E.) and Grant-in-aid (Kaken) from MEXT (K.E.). Preparation of platelets from hiPSCs were supported by Project of realization of regenerative medicine (phase II) from MEXT, Japan (K.E.) and A-STEP grant from Japan Science Technology (JST) Agency (H.N. and K.E).

\section{Author Contributions}


Y.N. and R.T. designed the experiments and performed experiments. S.N., H.E., and T.D. prepared samples. M.N. prepared materials. F.A. and T.F. supervised and designed the construction of a bioreactor. H.N. provided valuable discussion. K.E. wrote and edited the manuscript.

\section{Conflict of Interest}

H. Nakauchi and K. Eto are scientific advisory board of Megakaryon Co. Ltd. without salary. Others have no conflict of interest. 


\section{Figure legends.}

\section{Figure 1. Fabrication process of a sheet-like scaffold and Medium circulation model of}

\section{bioreactor.}

(A) (a) Produce a wax (made of toluenesulfonamide) model. (b) Wax model was dipped into PVA (Polyvinylalcohol) solution and pulled up with a dip coater (pull-up velocity: $1 \mathrm{~mm} / \mathrm{s}$ ).Dip coating of PVA onto the wax model was repeated 6 times, and each coating was followed by drying at room temperature for 30 minutes. (c) After an interval of 12 hours, the PVA-coated wax model was soaked in acetone. Because of the insolubility of PVA and solubility of wax in acetone, the wax was selectively dissolved, leaving the structure of PVA. (d) The PVA model then was coated with the polymer and $\mathrm{NaCl}$ particle solution using the dip coater (velocity: 3 $\mathrm{mm} / \mathrm{s})$. To coat the PLCL (poly (L-lactide-co-epsilon-caprolactone)) membrane as uniformly as possible, dip coating of polymer solution onto the PVA model was repeated three times in one direction and three times in the opposite direction by setting the model upside down. (e) Dip coated PLCL of the second layer in the same way as (d). (f) Each coating was followed by drying at room temperature for 2 minutes. The PLCL-coated PVA models then were soaked in deionized (DI) water followed by dissolution of PVA and the $\mathrm{NaCl}$ microparticles. After PVA and the $\mathrm{NaCl}$ microparticles were completely removed from the PLCL, the salt-leached PLCL scaffold was dried for 24 hours at room temperature.

(B) Culture within the reactor system was performed at $37^{\circ} \mathrm{C}$ in a $\mathrm{CO}_{2}$ incubator. Circulation was achieved using a PFA tube connected to a roller pump to produce flow. The left side schematic shows the cross-sectional view of bioreactor. Human umbilical vascular endothelial cells (HUVECs) were pre-applied for stable adhesion to the micropores in the scaffold 2 days 
before the megakaryocytes (MKs) were applied.

Figure 2. Confocal microscope images of megakaryocytes $\quad$ (A) Static culture. $\quad$ (B) Flow culture in which the shear stress produced with the reactor system depicted in Figure 2 was 0.14 dyne $/ \mathrm{cm}^{2}$. Nuclei were stained with DAPI (blue) and CD41a was stained with anti-human CD41a-FITC (green). Low-magnification images (left top) shows only DAPI staining. Bars, $50 \mu \mathrm{m}$.

Figure 3. Original model of the bioreactor and comparison of platelet yield (static vs. reactor) using the bioreactor system.

(A) The bioreactor was $25 \mathrm{~mm}$ x $28 \mathrm{~mm}$ and contained numerous circuits for flow. The termination of each circuit was a pore $(\sim 8 \mu \mathrm{m})$ formed by a pocket containing a single MK. MKs were stimulated to mature by the pressure flow, while platelet release was stimulated by the main flow. (B) Numbers of released platelets were determined based on CD41a ${ }^{+} \mathrm{CD} 42 \mathrm{~b}^{+}$ counts per reservoir (over a period of $12 \mathrm{~h}$ ). Black bars show the platelet counts obtained under static conditions using the protocol described in ref. 14. Gray bars show the platelet counts obtained using the first generation bioreactor. Number of platelets from static condition equalized 1.0. The input was $1.2 \times 10^{5}$ mature MKs in both conditions.

Figure 4. Confocal micrographs showing the time course of a platelet release from a MK (green: CD41a, blue: Hoechst). Nuclei were stained with Hoechst. 
Figure 5. Schematic of an improved bioreactor with two-directional flow (second type of a bioreactor) and comparison of platelet yields (static vs. reactor).

(A) The illustration shows the design of a bioreactor with two-directional flow in which the angle between the directions of the main and pressure flow is $60^{\circ}$. Platelets were generated from MKs placed between the slits. The slits were $16 \mu \mathrm{m}$ wide with $4 \mu \mathrm{m}$ in between.

(B) Numbers of released platelets were determined based on hESC-derived CD41a ${ }^{+} \mathrm{CD} 42 \mathrm{~b}^{+}$ platelet population counts per reservoir (over a period of $12 \mathrm{~h}$ ). Black bars show the platelet counts obtained under static conditions using the protocol described in ref. 14. Gray bars show the platelet counts obtained using second generation of a bioreactor. Average number of platelets from static condition equalized 1.0. The input was $1.2 \times 10^{5}$ mature MKs in both conditions. Experiments were mean+/-s.e.m. (n=4). (C) Representative dot plots of platelets in flow cytometer, derived from human ESCs through second generation of a bioreactor (right). Same gate in side scatter (SSC) and forward scatter (FSC) of fresh human platelets was used for ESC-derived platelets in flow cytometer (left). This gate was re-analyzed for detection of CD41a (integrin $\alpha \mathrm{IIb}$ ) and CD42b (GPIb $\alpha$ ). (D) Purity of CD41a ${ }^{+}$or CD41a $\mathrm{a}^{+} \mathrm{CD} 42 \mathrm{~b}^{+}$platelet population of gated population depicted in the left panel of Figure 5C (SSC and FSC) was also evaluated as comparison between static culture condition and bioreactor. Black column indicates static culture and silver column indicates bioreactor. In each column, white bar is merged as $\mathrm{CD} 41 \mathrm{a}^{+} \mathrm{CD} 42 \mathrm{~b}^{+}$population.

Figure 6. Integrin activation of human ESC- or iPSC-platelets. Integrin activation in ESC-platelets (KhES-3) (A) and in iPSC-platelets (TkDA3-4)(B). The binding of PAC-1 
(indicative of platelet activation) to individual platelets was quantified in the absence and presence of $50 \mu \mathrm{M}$ ADP, 0.5 Unit human Thrombin, or $200 \mathrm{nM}$ phorbol myristate acetate (PMA) using flow cytometry. Mean fluorescence intensity (MFI) of bound PAC-1, obtained from $\mathrm{CD} 42 \mathrm{~b}^{+}$fraction was evaluated (experiments were independently performed twice). 
Figure 1 Nakagawa et al.

A

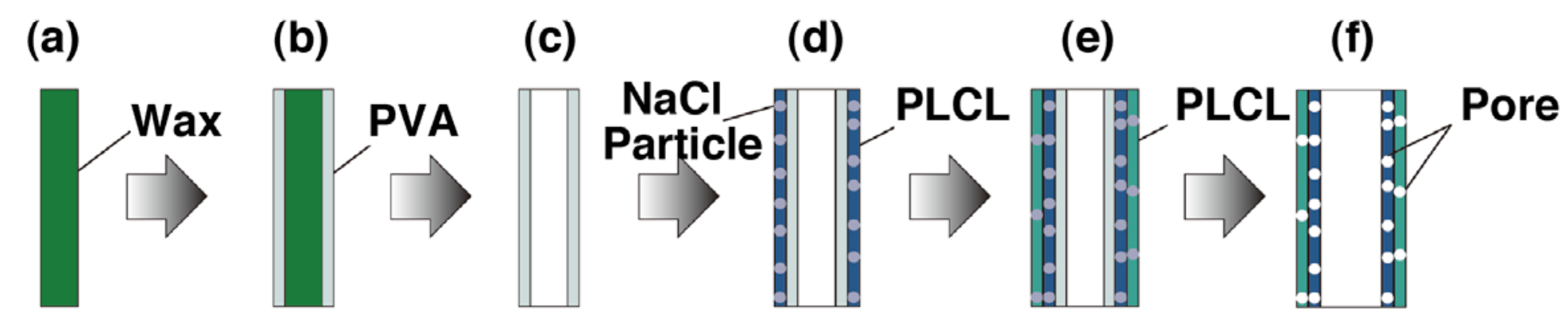

B

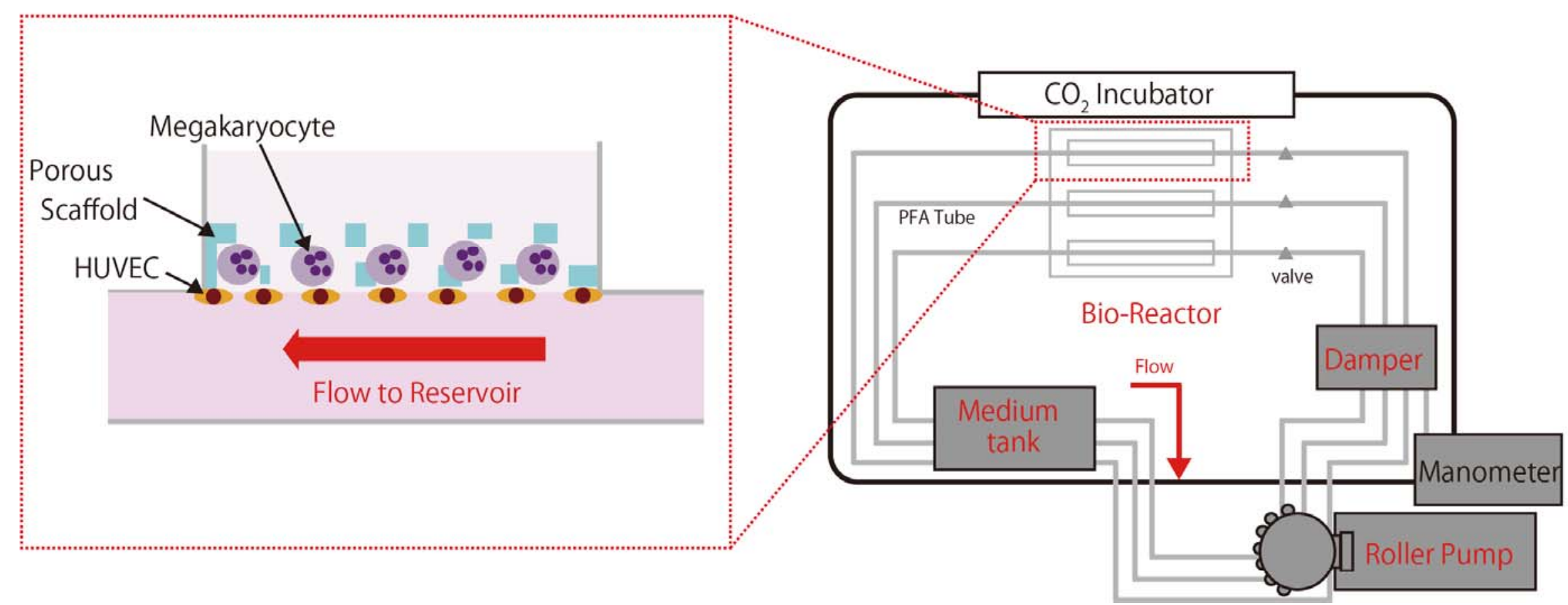


Figure 2 Nakagawa et al.
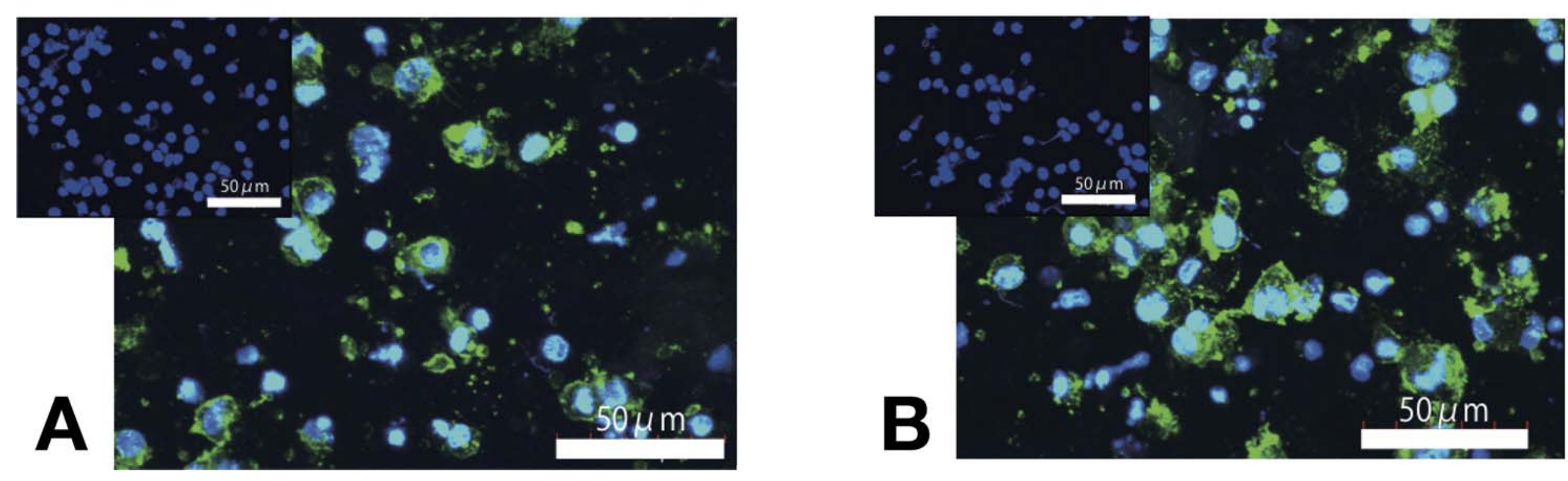
Figure 3 Nakagawa et al.

A

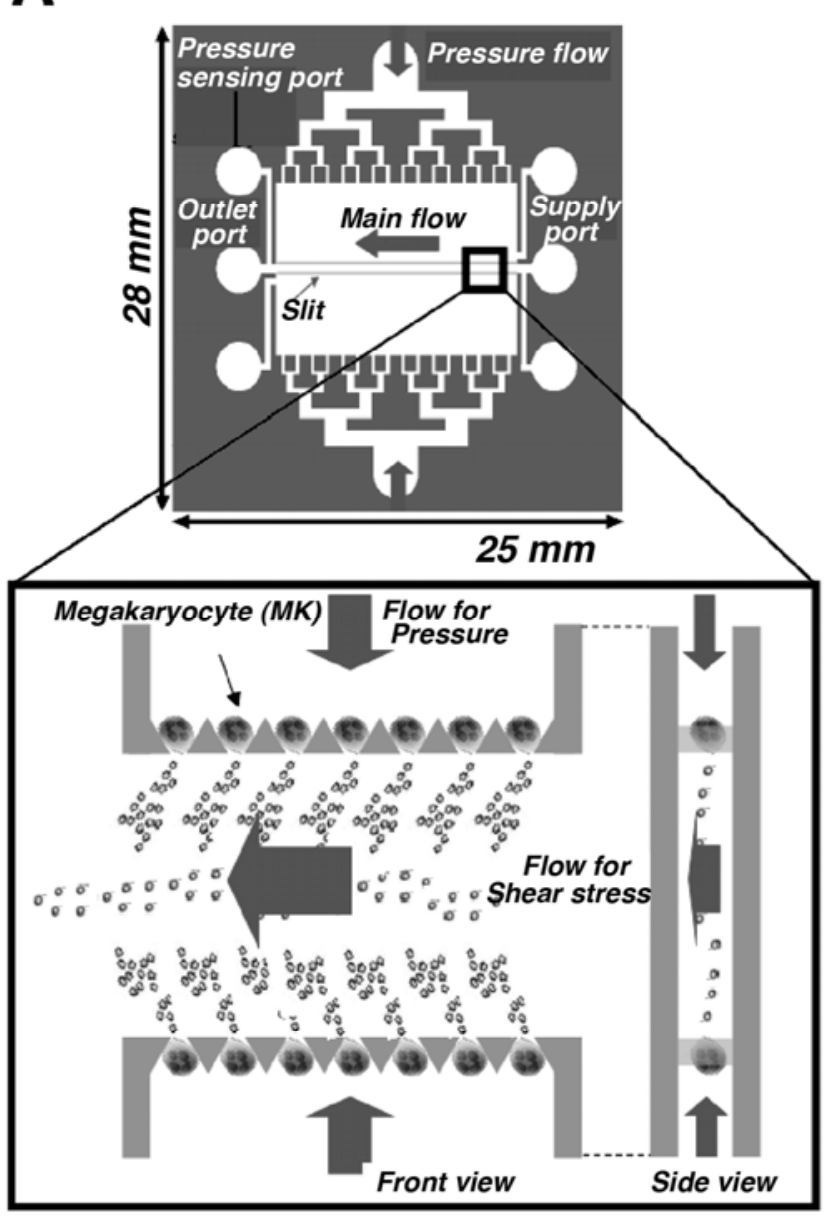

B

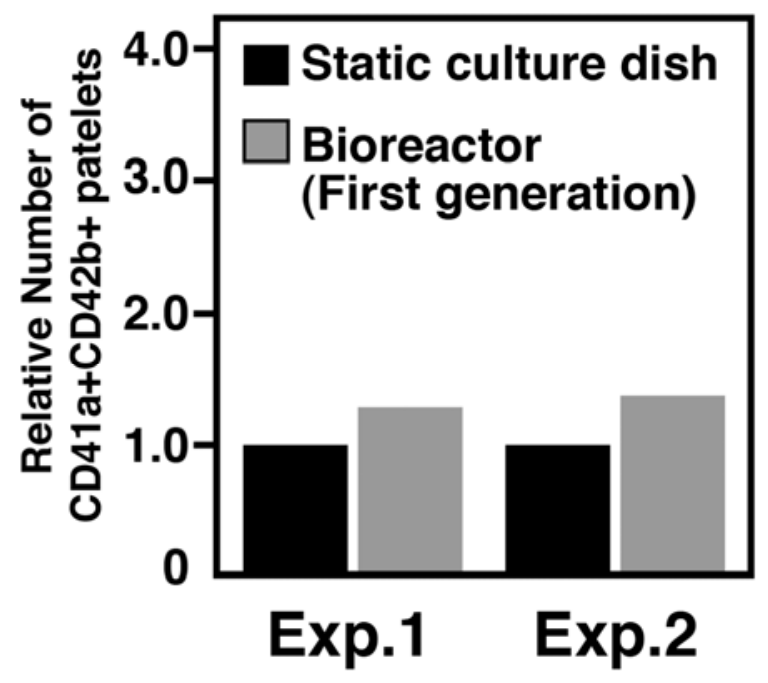


Figure 4 Nakagawa et al.

\section{$0 \mathrm{~min}$}

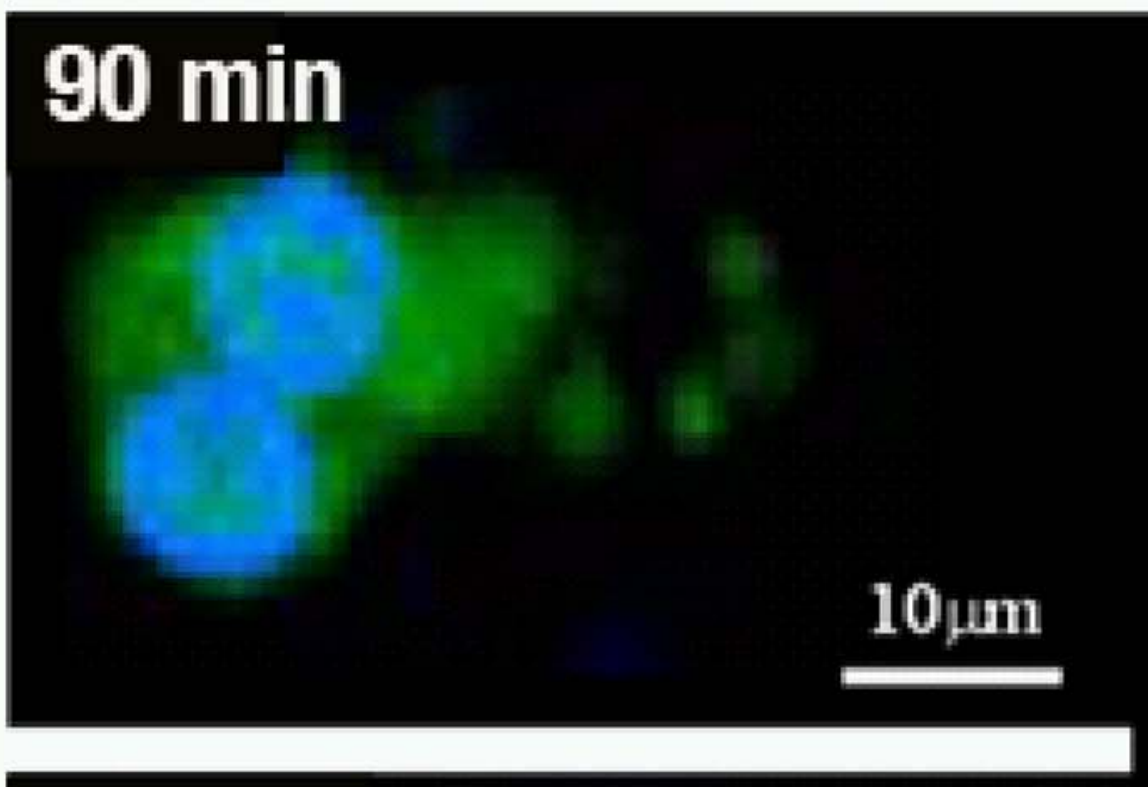

$92 \mathrm{~min}$

$93 \mathrm{~min}$ 
A

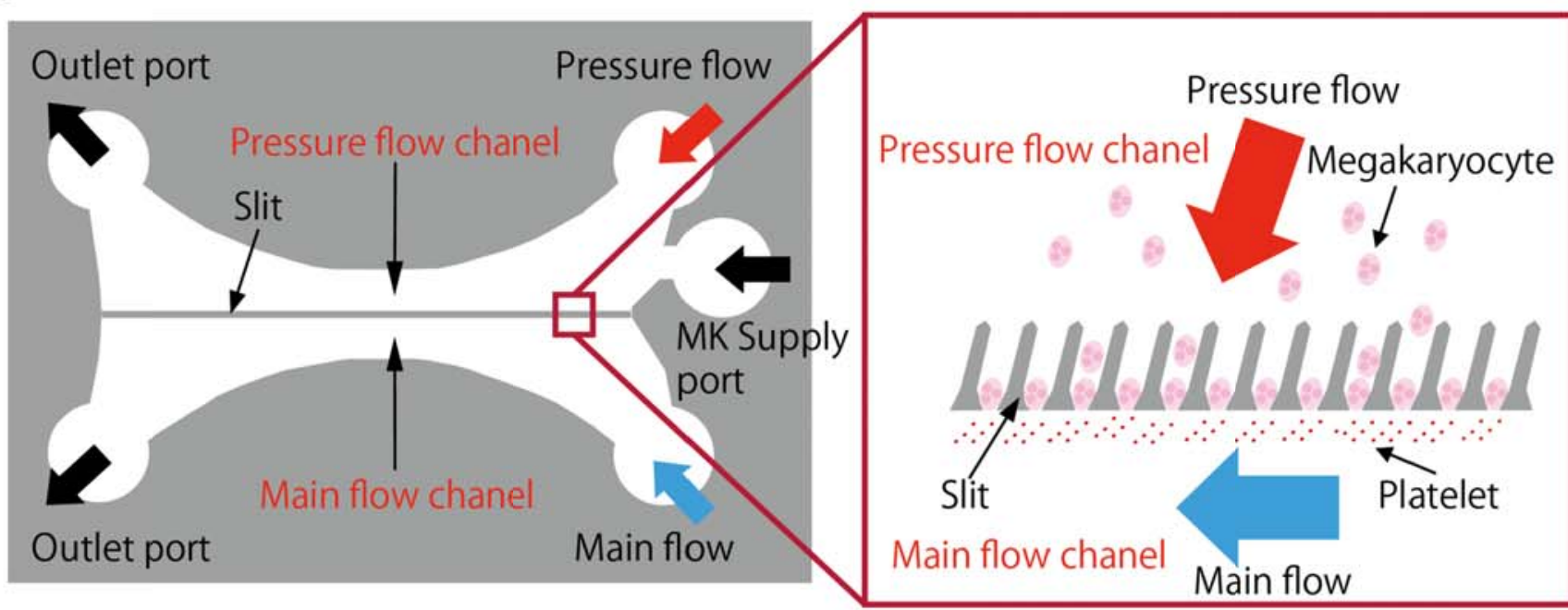

B

D

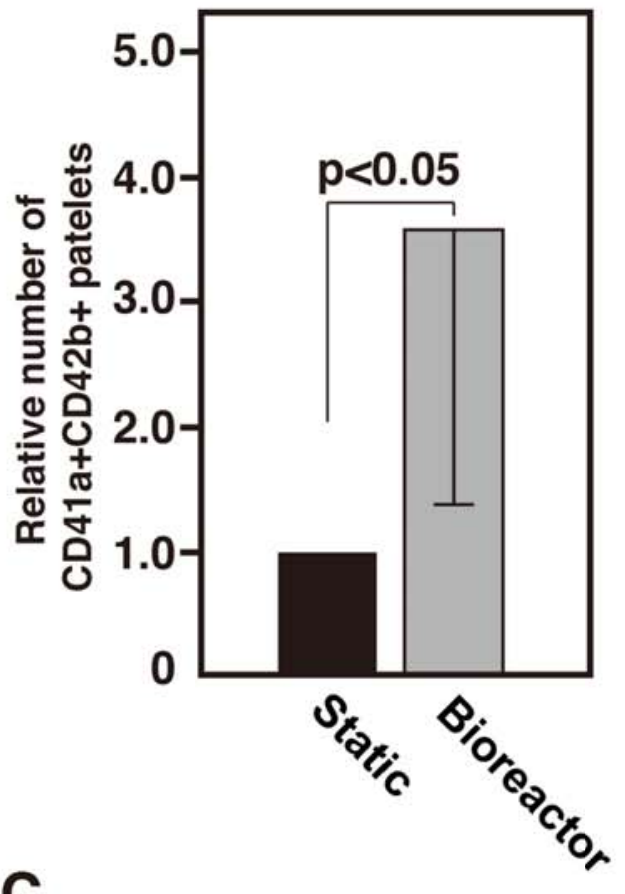

Peripheral blood

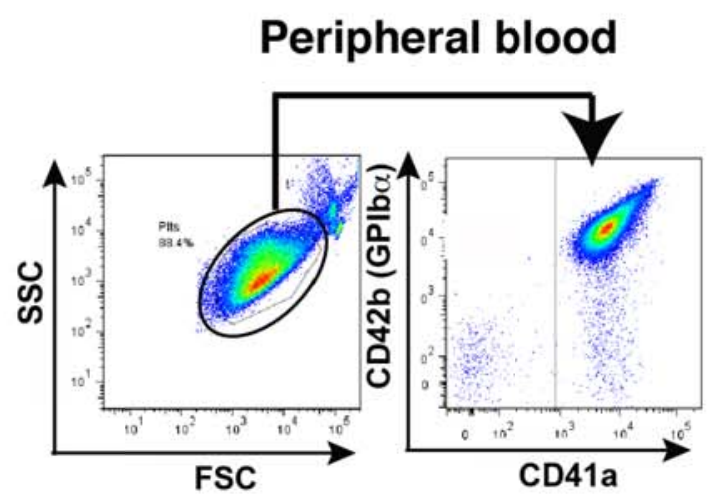

C

Figure 5 Nakagawa et al.

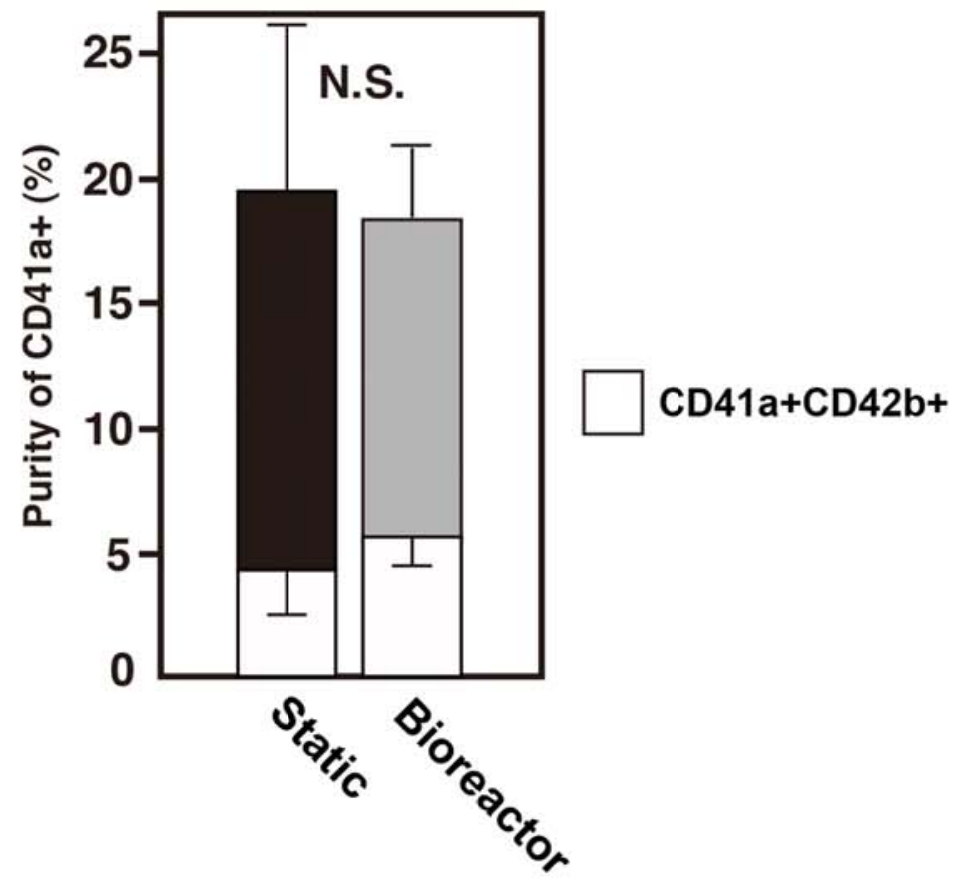

hESC-platelets from bioreactor

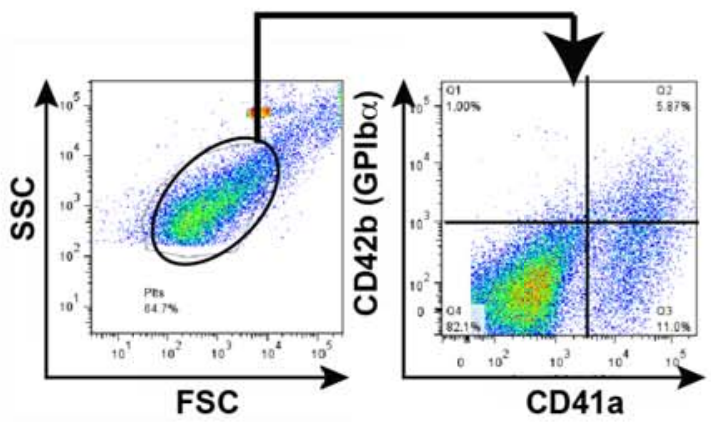


Figure 6 Nakagawa et al.
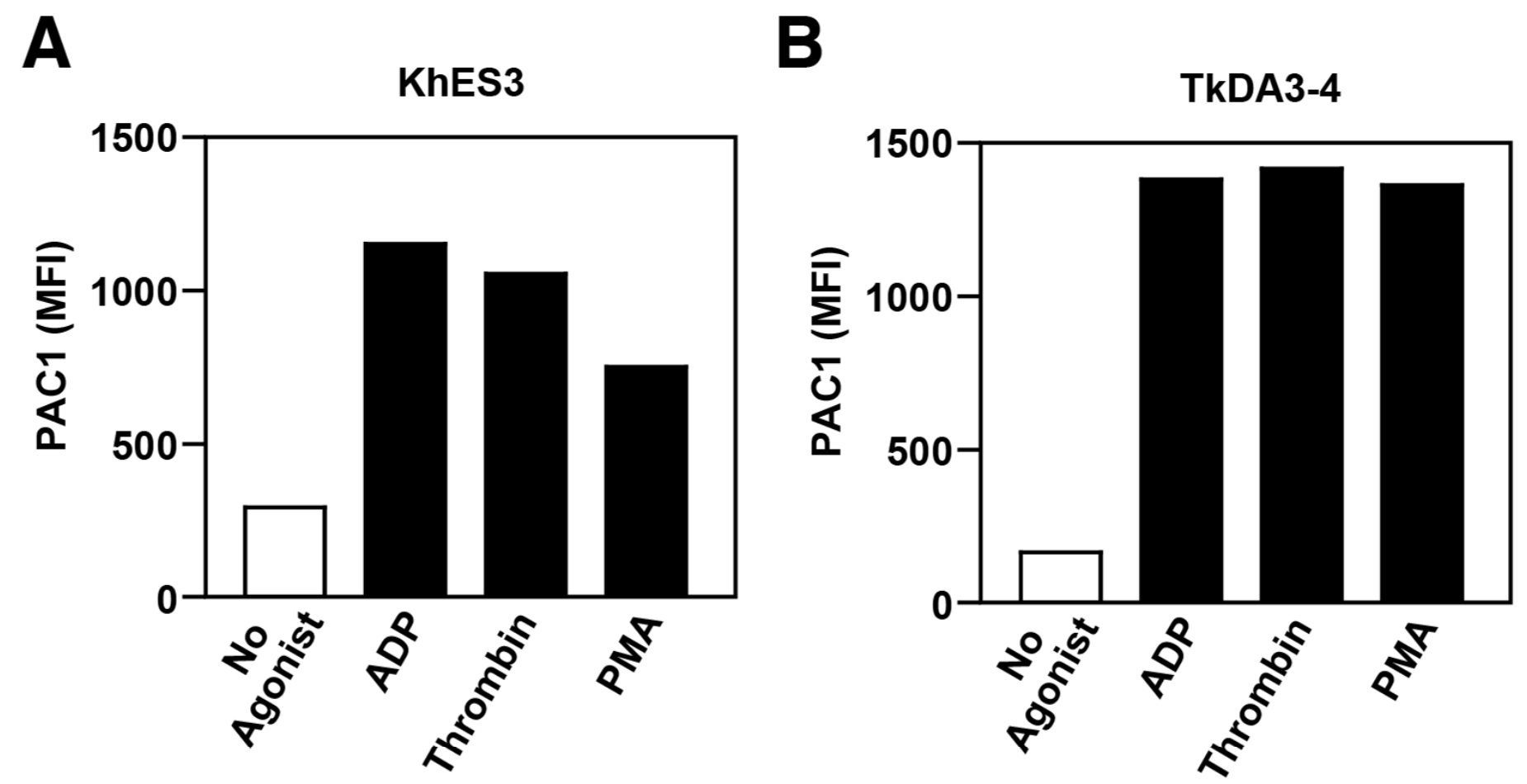
Table 1: Solution used in Salt-Leaching

\begin{tabular}{|c|c|c|}
\hline & Solution 1 & Solution 2 \\
\hline Diameter of NaCL & $\begin{array}{c}\text { Under } \\
23[\mu \mathrm{m}]\end{array}$ & $50-106[\mu \mathrm{m}]$ \\
\hline $\mathrm{NaCl}: \mathrm{PLCL}$ & $20: 1$ & $10: 1$ \\
\hline Chloroform:PLCL & $100: 3$ & $100: 3$ \\
\hline
\end{tabular}

